\title{
TEMA-2017: Proteína Ligadora de Ácidos Grasos Intestinal en Neonatos con Enterocolitis Aguda Necrotizante
}
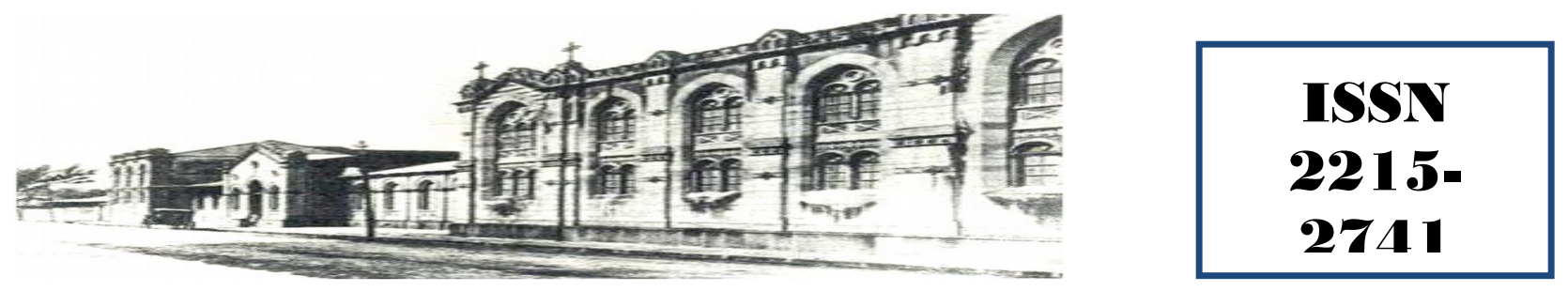

Haspital San quan de Dias, San Jasé, Casta Rica. Fundada en 1845

Recibido:

$06 / 12 / 16$

Aceptado:

$15 / 01 / 17$

Jasson Rojas Vega ${ }^{1}$

${ }^{1}$ Médico Residente de la Especialidad de Cirugía Cardiovascular, Hospital Calderón Guardia. Universidad de Costa Rica. Correo electrónico: jirrvzafa@gmail.com

\section{RESUMEN}

La enterocolitis aguda necrotizante (EAN) es la principal emergencia abdominal neonatal en Unidades de Cuidados Intensivos, representando además la principal causa de muerte gastrointestinal en estos pacientes. El diagnóstico de basa en criterios clínicos, de laboratorio y radiológicos que en muchos ocasiones pueden ser inespecíficos, de tal forma que es necesario el desarrollo de métodos diagnósticos más certeros. La presente revisión evalúa la utilidad diagnósticos y pronóstica de la proteína ligadora de ácidos grasos intestinal (I-FABP) en pacientes con EAN.

\section{PALABRAS CLAVE}

Enterocolitis aguda necrotizante. Proteína ligadora de ácidos grasos intestinal. Neonatos. Pediatría. Unidad de cuidados intensivos.

\section{ABSTRACT}

Acute necrotizing enterocolitis is the main neonatal abdominal emergency in Intensive Care Units, and represents the leading cause of gastrointestinal death in these patients. The diagnosis is based on clinical, laboratory and radiological findings which in many cases may be nonspecific, so that is needed development of more accurate diagnostic methods. This review evaluates the diagnostic and prognostic utility of intestinal fatty acid binding protein (I- FABP) in patients with acute necrotizing enterocolitis.

\section{KEY WORDS}

Acute necrotizing enterocolitis. Intestinal fatty acid binding protein. Neonates. Paediatrics. Intensive care unit.

\section{INTRODUCCIÓN}

La EAN es un desorden inflamatorio severo del tracto gastrointestinal que afecta aproximadamente al $1 \%$ de los pacientes en Unidad de Cuidado Intensivo Neonatall. Tiene 
mayor incidencia en neonatos prematuros ${ }^{(2)}$, y se estima que su incidencia es de $7 \%$ en neonatos con muy bajo peso al nacer. ${ }^{(3)}$

Representa la principal causa de muerte por enfermedad gastrointestinal en neonatos pretérmino, y se caracteriza por necrosis de la pared intestinal, sepsis sistémica y falla multiorgánica. (2) Es un patología con una mortalidad de aproximadamente $20-40 \%$ (4), especialmente en pacientes que requieren cirugía.

(5) Los sobrevivientes sufren de secuelas severas como problemas de alimentaciones, falla para progresar, dependencia de nutrición parenteral, síndrome de intestino corto $\mathrm{y}$ alteraciones del neurodesarrollo. ${ }^{(6)}$

La presente revisión pretende determinar las propiedades diagnósticas de la I-FABP tanto a nivel sanguíneo como urinario para la EAN, y su posible utilidad como marcador de severidad de la enfermedad, ya sea para definir la necesidad de resolución quirúrgica y la extensión de la necrosis intestinal.

\section{DISCUSIÓN}

\section{Fisiopatología}

La patogénesis exacta aún se desconoce, pero la principal hipótesis establece una alteración de la respuesta inflamatoria por parte del intestino hacia las baterías luminales. Se genera una lesión epitelial iniciada por diferentes eventos, incluyendo isquemia intestinal, alimentación con fórmula y colonización por patógenos oportunistas, lo que lleva a activación del sistema inmune innato de la mucosa intestinal. ${ }^{(7)}$

\section{Diagnóstico}

$\mathrm{Su}$ diagnóstico se realiza por medio de una combinación de hallazgos clínicos, de laboratorio y radiológicos divididos en signos sistémicos, abdominales y radiológicos según los criterios modificados de Bell. ${ }^{(8)}$

\section{Tratamiento}

El tratamiento inicial consiste en suspender la vía oral, colocación de sonda nasogástrica, antibióticos intravenosos de amplio espectro y soporte cardiopulmonar ${ }^{(9)}$, y en caso de deterioro clínico a pesar de tratamiento médico máximo o perforación intestinal, el tratamiento de elección es la resección del intestino necrótico. ${ }^{(10)}$

\section{Características clínicas}

Las características clínicas en la presentación de la enfermedad son poco específicas. Signos constitucionales presentes al inicio de la enfermedad son difíciles de distinguir de otras condiciones neonatales, como la dismotilidad gastrointestinal del prematuro o el íleo intestinal inducido por sepsis. ${ }^{(5)}$ Las radiografías y el ultrasonido de abdomen pueden confirmar el diagnóstico en estadíos avanzados de la enfermedad ${ }^{(11)}$, sin embargo los signos radiológicos tempranos son difíciles de interpretar.

La neumatosis intestinal tiene poca sensibilidad para el diagnóstico, con baja concordancia en su interpretación incluso entre radiólogos pediátricos12. El pneumoperitoneo como indicador de cirugía puede estar ausente hasta en un $50-60 \%$ de los pacientes perforados. ${ }^{(5)}$

El hecho de que los signos, síntomas, laboratorios y estudios radiológicos sean inespecíficos en estadios iniciales de la enfermedad hace el diagnóstico difícil ${ }^{(13)}$, por lo que se requiere de otros parámetros diagnósticos para el manejo de los pacientes con EAN.

Los criterios diagnósticos al inicio de la enfermedad son muy amplios, por lo que sería ideal identificar aquellos neonatos que van a desarrollar definitivamente la enfermedad tanto para prevenir intervenciones innecesarias como para establecer medidas terapéuticas adecuadas. (12) Por ejemplo, la intervención quirúrgica temprana podría llevar a laparotomías innecesarias, mientras que postergarla puede llevar a progresión rápida de la enfermedad, sepsis y muerte. ${ }^{(5)}$

En vista de la alta mortalidad y morbilidad posterior a la perforación de la pared intestinal, la detección temprana de necrosis antes de la perforación podría potencialmente mejorar el pronóstico $^{(5)}$, por lo que la detección temprana de necrosis intestinal, especialmente en ausencia de perforación, es un problema fundamental para determinar el tiempo adecuado para la cirugía. ${ }^{(9)}$ 
La EAN usualmente tiene una evolución rápida, con pocos o casi ningún signo que se pueda usar para predecir los resultados, por lo que su rápido establecimiento y progresión hasta la muerte, así como su morbilidad severa si el paciente sobrevive, requieren de herramientas diagnósticas tempranas que se puedan usar para determinar aquellos niños en mayor riesgo de progresión de la enfermedad, y aquellos donde haya medidas preventivas que se puedan aplicar (14), por lo que se necesita de marcadores serológicos con adecuada sensibilidad y especificidad para el diagnóstico temprano de esta enfermedad. ${ }^{(12)}$

Con el daño de la mucosa intestinal hay liberación de proteínas citoplasmáticas de los enterocitos hacia la circulación, las cuales se consideran marcadores de daño de la pared intestinal. ${ }^{(2)}$ Las proteínas ligadoras de ácidos grados son proteínas pequeñas, con un peso molecular de aproximadamente $14-15 \mathrm{kDa}$, que constituyen el $2 \%$ de las proteínas del citosol de los enterocitos maduros. Son parte de una familia de 9 proteínas ligadoras responsables del transporte de ácidos grasos de cadena larga. ${ }^{(2)}$ Datos preliminares muestran que las propiedades diagnósticas de estas proteínas para el diagnóstico temprano de EAN son muy prometedoras. ${ }^{(7)}$

\section{I-FABP}

La I-FABP es una proteína citoplasmática hidrosoluble15, con alta sensibilidad orgánica ya que se sintetizada solamente en los enterocitos5, localizada en la cima de las vellosidades intestinales9, con su máxima expresión a nivel del yeyuno distal16. Otras proteínas, como la proteína ligadora de ácidos grasos hepática, la cual es sintetizada en los enterocitos y los hepatocitos, y el factor trefoil 3, que se expresa en las células caliciformes intestinales y en las células epiteliales productoras de mucina en múltiples partes del cuerpo5, también han sido utilizadas como marcadores de daño intestinal.

Cuando ocurre daño de la pared intestinal en EAN, se produce liberación de la I-FABP es en la circulación9, y debido a su pequeño tamaño puede pasar a través del aparato glomerular y ser eliminada en la orina17. Así, se puede medir en sangre y en orina18. Sin embargo, una limitación de esta proteína es su baja disponibilidad en el uso clínico diario 7 .

\section{Niveles Sanguíneos}

Evennett et al ${ }^{(12)}$ realizaron un metanálisis de 3 estudios de cohorte donde se utilizaba la utilidad de la I-FABP en sangre para el diagnóstico de EAN, con un total de 51 pacientes, con los cuales se determinó una sensibilidad de $62 \%$ y una especificidad de $100 \%$.

De esta forma, esta proteína es un marcador específico pero poco sensible para el diagnóstico de enterocolitis. Sin embargo el punto de corte diagnóstico aún está por determinarse ya que se utilizaron diferentes valores: en el estudio de Guthman et al se utilizó $5 \mathrm{ng} / \mathrm{ml}$, mientras que en los estudios de Edelson et al y Lieberman et al se utilizó $1,87 \mathrm{ng} / \mathrm{ml}$.

En el estudio prospectivo realizado por Benkoe et al ${ }^{(7)}$, se comparó los niveles séricos de la proteína de ácidos grasos intestinal en 15 pacientes con EAN diagnosticada según los criterios modificados de Bell al inicio de la enfermedad, con los niveles séricos en 14 controles sanos, menores de 6 meses, peso al nacer menor a $2000 \mathrm{~g}$, con edad gestacional, peso al nacer y edad al diagnóstico similar al paciente enfermo.

Se encontró que los niveles séricos de la proteína fueron significativamente mayores (el doble) en pacientes con EAN en comparación con el grupo control $(2.8 \mathrm{ng} / \mathrm{ml}$ vs $1.4 \mathrm{ng} / \mathrm{ml}$ respectivamente). Los pacientes con enterocolitis fueron divididos en dos grupos: el de manejo médico, con 5 pacientes, y el de manejo quirúrgico, con 10 pacientes.

Los niveles de la proteína fueron significativamente mayores en pacientes tratados quirúrgicamente en comparación con los controles $\quad(4.2 \mathrm{ng} / \mathrm{ml} \quad$ vs $\quad 1.4 \mathrm{ng} / \mathrm{ml}$ respectivamente), pero no hubo diferencias significativas entre los pacientes tratados medicamente y los tratados quirúrgicamente $(2.2 \mathrm{ng} / \mathrm{ml}$ vs $4.2 \mathrm{ng} / \mathrm{ml}$ respectivamente), sin embargo, hubo una tendencia hacia valores mayores en pacientes que fueron operados en este estudio. 
En el estudio realizado por $\mathrm{Ng}$ et al ${ }^{(5)}$, se evaluó el uso de tres proteínas de la barrera intestinal, la I-FABP, la proteína ligadora de ácidos grasos hepática $y$ el factor trefoil 3, como biomarcadores para diferenciar EAN de pacientes sanos y de pacientes sépticos, además de identificar a los pacientes más severos (aquellos que requieren cirugía contra aquellos que no la requieren, y aquellos con mayor mortalidad).

En el grupo con EAN hubo 20 pacientes (12 tratados quirúrgicamente y 8 tratados médicamente), en el grupo con septicemia hubo 40 pacientes, y en el grupo control hubo 40 pacientes. Para investigar el efecto combinado de los 3 biomarcadores, se creó el "LIT Score" (por sus siglas en inglés: L-FABP, I-FABP y TFF3), como una escala desde 0 hasta 9 , tomando en cuenta de la concentración plasmática de cada biomarcador, se le asigna un valor de 0 hasta 3, dependiendo de la siguiente tabla:

\begin{tabular}{cccc}
\hline & I-FABP & L-FABP & TFF3 \\
\hline $\mathbf{0}$ & $<0.8$ & $<70 \mathrm{ng} / \mathrm{mL}$ & $<4 \mathrm{ng} / \mathrm{mL}$ \\
& $\mathrm{ng} / \mathrm{mL}$ & & \\
$\mathbf{1}$ & $0.8-4.4$ & $70-235$ & $4-9.6$ \\
& $\mathrm{ng} / \mathrm{mL}$ & $\mathrm{ng} / \mathrm{mL}$ & $\mathrm{ng} / \mathrm{mL}$ \\
$\mathbf{2}$ & $4.4-23$ & $235-650$ & $9.6-16$ \\
& $\mathrm{ng} / \mathrm{mL}$ & $\mathrm{ng} / \mathrm{mL}$ & $\mathrm{ng} / \mathrm{mL}$ \\
$\mathbf{3}$ & $>23 \mathrm{ng} / \mathrm{mL}$ & $>650 \mathrm{ng} / \mathrm{mL}$ & $>16 \mathrm{ng} / \mathrm{mL}$ \\
\hline
\end{tabular}

Tabla 1: Cuadro 1. Niveles de I-FABP, L-FABP y TFF3 para el LIT Score.

Fuente: $\mathrm{Ng}$ et al. (2013). Gut-associated biomarkers L-FABP, I-FABP, and TFF3 and LIT Score for diagnosis of surgical necrotizing enterocolitis in preterm infants. Annals of Surgery, 258, 1111-1118.

Con respecto a los niveles plasmáticos, los niveles de los 3 biomarcadores y el LIT Score fueron significativamente mayores en el grupo de EAN en comparación con el grupo de paciente con septicemia y el grupo de pacientes control. Esto establece una correlación positiva entre estos biomarcadores y el LIT Score, y pacientes con EAN, por lo que las concentraciones séricas de estos están directamente relacionadas con daño de la mucosa intestinal, y no se deben al síndrome de respuesta inflamatoria sistémica. Todos los biomarcadores tuvieron una sensibilidad de al menos $50 \%$ y una especificidad de al menos $95 \%$ para diferenciar pacientes con EAN de pacientes sépticos 0 controles.

Con respecto a la severidad de la enfermedad, se observaron niveles significativamente mayores de los 3 biomarcadores y del LIT Score en pacientes con EAN quirúrgica que en casos no quirúrgicos, siendo el LIT Score el que tiene mejor certeza diagnóstica. Un LIT Score mayor de 4.5 mostró una sensibilidad de un $83 \%$ y una especificidad de un $100 \%$ para diferenciar casos quirúrgicos de no quirúrgicos, mientras que cada biomarcador por separado mostró una sensibilidad de al menos $67 \%$ y una especificidad de al menos $75 \%$ para este mismo propósito.

Con respecto a la mortalidad, los niveles de los 3 biomarcadores y el LIT Score fueron significativamente mayores en los pacientes que murieron que en los que sobrevivieron, un LIT Score $>6$ mostró una sensibilidad de $78 \%$ y una especificidad de $91 \%$ para muerte en pacientes con EAN.

De esta forma, estos biomarcadores se asocian con EAN quirúrgica, siendo el LIT Score el mejor en identificar pacientes quirúrgicos $\mathrm{y}$ con mayor mortalidad en estadíos tempranos de la enfermedad. Sin embargo, existe traslape entre las concentraciones bajas-moderadas de los marcadores en EAN no quirúrgica y casos de sepsis o controles, de tal forma que estos biomarcadores no son tan útiles en identificar casos leves de la enfermedad.

Se cree que algunos pacientes con sepsis pueden tener elevaciones moderadas de estos marcadores probablemente asociado con traslocación bacteriana. Estos resultados son importantes ya que se pueden detectar casos más severos al inicio de la enfermedad, ya sea los que requieran cirugía o los que tengan mayor probabilidad de morir.

Aydemir et al ${ }^{(14)}$ evaluaron los niveles de la IFABP al inicio de la enfermedad, a las 24 horas y a las 72 horas, en 41 pacientes con sospecha de EAN, basados en signos abdominales, sistémicos y radiológicos, contra los niveles en 31 pacientes control admitidos en una Unidad de Cuidados Intensivos Neonatal con peso y edad gestacional 
semejante a los casos, sin EAN, sepsis, síndrome de respuestas inflamatoria sistémica ni errores congénitos del metabolismo. Los casos se dividieron en 3 grupos: grupo 1 (estadío I de la enfermedad), grupo 2A (estadío II de la enfermedad) y grupo 2B (estadío III de la enfermedad).

Los niveles iniciales promedio en cada grupo fueron los siguientes: en el grupo 1 de $324.0 \pm$ $165.8 \mathrm{pg} / \mathrm{ml}$, en el grupo 2 de $764.7 \pm 465.1$ $\mathrm{pg} / \mathrm{ml}$, en el grupo 3 de $360.2 \pm 463.5 \mathrm{pg} / \mathrm{ml}$, y en el grupo control de $76.9 \pm 115.9 \mathrm{pg} / \mathrm{ml}$. Los niveles en el grupo control fueron significativamente menores en los pacientes control con respecto a los otros grupos de pacientes con la enfermedad.

Para definir un valor diagnóstico a las $72 \mathrm{~h}$ en el grupo de casos, un nivel de I-FABP $>116 \mathrm{pg} / \mathrm{ml}$ tiene una sensibilidad de $59.9 \%$ y una especificidad de 95\% para el diagnóstico de EAN. Si se analiza solo el grupo de paciente con la enfermedad, no hubo diferencias significativas en los niveles de la proteína entre los pacientes que sobrevivieron y los que murieron, además de que no hubo diferencias significativas en los niveles de la proteína entre los casos quirúrgicos y los no quirúrgicos al inicio de la enfermedad ni a las 24 horas, sin embargo, los niveles a las 72 horas fueron significativamente mayores en los pacientes quirúrgicos.

Este estudio encontró niveles mayores de la proteína en estadíos II y III que en el estadío I. además, se observaron niveles mayores de la proteína a las 72 horas del inicio de la enfermedad en pacientes con estadio III de la enfermedad. Los niveles en los estadíos I y II disminuyeron progresivamente desde el inicio de la enfermedad hasta las 72 horas, mientras que en el estadío 3 aumentaron de las 24 a las 72 horas.

Heida et al ${ }^{(9)}$ realizaron un análisis de los niveles séricos de la I-FABP en dos estudios de cohorte, para un total de 19 casos, en pacientes con EAN manejada de manera quirúrgica, y los correlacionaron con el tamaño del intestino resecado durante laparotomía. En ambos cohortes, los niveles plasmáticos al inicio de la enfermedad se correlacionaron significativamente con el tamaño del intestino resecado, sin embargo no hay diferencias significativas ente los niveles en pacientes con resecciones del intestino delgado y pacientes con resecciones del colon.

De esta forma concluyen que los niveles plasmáticos de la I-FABP se correlacionan con el tamaño del intestino resecado en neonatos con EAN quirúrgica. Sin embargo, este estudio cuenta con varias limitaciones: el tamaño de la muestra es pequeño, los tiempos entre los síntomas y la cirugía en ambos cohortes fueron diferentes, y se utilizaron diferentes equipos de ELISA para medir la proteína en ambos en ambos cohortes.

\section{Niveles Urinarios}

En el estudio realizado por Evennett et al ${ }^{(19)}$, se compararon los niveles urinarios de la I-FABP en paciente con EAN con pacientes controles (edad gestacional y peso semejantes, ingresados en Unidad de Cuidados Intensivos Neonatal, sin EAN, sin sepsis o shock séptico, sin síndrome de respuesta inflamatoria sistémica y sin errores congénitos del metabolismo). En el grupo de paciente con enterocolitis hubo 16 pacientes (4 manejados médicamente y 12 manejas quirúrgicamente), y en el grupo control hubo 6 pacientes. Debido a variaciones en el método de recolección de la muestra de orina, los datos se normalizaron y expresaron en una relación IFABP entre creatinina (I-FABP/Cr), en picogramos por milimoles.

En todos los controles la relación I-FABP/Cr urinaria fue $<2 \mathrm{pg} / \mathrm{mmol}$, lo que se ha sugerido como el punto de corte para distinguir neonatos con EAN de neonatos sin la patología. En los neonatos con EAN quirúrgica la relación I$\mathrm{FABP} / \mathrm{Cr}$ urinaria fue $>2 \mathrm{pg} / \mathrm{mmol}$ en 9 de 16 pacientes (56.2\%), y $<2 \mathrm{pg} / \mathrm{mmol}$ en los otros 7 de 16 pacientes $(43.7 \%)$. Hubo una tendencia hacia el aumento de la relación I-FABP/Cr urinaria al ingreso en pacientes con EAN, pero esto no alcanzó significancia estadística, además hubo una diferencia estadísticamente significativa en el pico de la relación I-FABP/Cr urinaria en el preoperatorio de pacientes que requirieron cirugía, sin embargo no hubo diferencias estadísticamente significativas en la relación I-FABP/Cr urinaria al ingreso. 
Neonatos con enfermedad más severa tuvieron niveles de I-FABP/Cr mayores que aquellos con enfermedad focal. Además, se espera que el tamaño del intestino resecado se correlacione con los niveles de I-FABP/Cr, sin embargo este análisis no se logró realizar en este estudios. Usando $2 \mathrm{pg} / \mathrm{mmol}$ como punto de corte, se tiene una sensibilidad del $58 \%$ y una especificidad del $100 \%$ para el diagnóstico de EAN.

Estos resultados sugieren que la relación IFABP/Cr por sí sola no es un marcador útil para diferenciar casos quirúrgicos de no quirúrgicos, se cree que esto es porque puede haber daño de la mucosa intestinal con liberación de I-FABP sin que haya necrosis transmural que requiera cirugía, por lo que un biomarcador de necrosis muscular intestinal sería más útil. Sin embargo, una vez se inicia el manejo quirúrgico la relación i-FABP/Cr aumenta, por lo que es más útil para diferenciar necrosis focal de necrosis más extensa.

A su vez, Gregory et al ${ }^{(18)}$ realizaron un estudio de cohorte en 70 neonatos prematuros que tuvieran niveles urinarios 7 días y 3 días previo al diagnóstico de EAN, comparándolos con los niveles en esos mismos días en pacientes control.

En los 70 casos la concentración de la I-FABP fue mayor en los casos de EAN que en los controles 7 días previo al diagnóstico. Estratificado por criterios de Bell, los niveles fueron significativamente mayores en los estadíos I y III de la enfermedad 7 días antes del diagnóstico. Para el análisis de los niveles urinarios 3 días previo a la presentación de la enfermedad se analizaron 49 casos y 49 controles, donde se vio que los niveles urinarios 3 días antes del diagnóstico fueron significativamente mayores en todos los estadíos de Bell.

Se encontró que una concentración urinaria de la I-FABP mayor a $13.3 \mathrm{ng} / \mathrm{ml}$ siete días previo al diagnóstico tiene una sensibilidad del $60 \%$ y una especificidad del $78 \%$, mientras que una concentración mayor a $13.9 \mathrm{ng} / \mathrm{ml}$ tres días previo al diagnóstico tiene una sensibilidad del $65 \%$ y una especificidad del 84\% para el diagnóstico de EAN. Este estudio muestra que los niveles urinarios de la I-FABP podrían ser un marcador predictor de EAN desde una semana previo a la presentación de la enfermedad, y es aún más predictivos tres días antes, sin embargo, combinado con otros biomarcadores se podría aumentar la sensibilidad y la especificidad diagnóstica.

En el estudio realizado por Heida et al mencionado anteriormente, se determinó que los niveles de la I-FABP a nivel urinario al inicio de la enfermedad se correlacionan significativamente con el tamaño del intestino resecado. Sin embargo, es importante recordar que este estudio cuenta con varias limitaciones significativas ya mencionadas anteriormente.

\section{Limitaciones}

Existen múltiples causas por las cuales los niveles de la proteína fijadora de ácidos grasos intestinal pueden ser bajos a pesar de daño de la mucosa intestinal:

- La producción de esta proteína cesa una vez iniciada la lesión intestinal ${ }^{(5)}$

- La proteína se encuentra fundamentalmente en los enterocitos maduros de las vellosidades, si ha ocurrido daño de la mucosa previamente puede que los enterocitos maduros ya se hayan perdido y que ya se haya excretado en la orina. ${ }^{(19)}$

- La vida media de la proteína ligadora de ácidos grasos hepática es de aproximadamente 11 minutos ${ }^{(20)}$, y se cree que la vida media de la proteína ligadora de ácidos grados intestinal ha se ser similar, de tal forma que es más probable detectarla si hay daño continuo de la mucosa intestinal. ${ }^{(19)}$ Si la lesión no es continua, podrían detectarse niveles bajos de la misma a pesar de la lesión.

- $\quad$ Puede que el área de necrosis sea en un segmento muy pequeño, o que la elevación sea transitoria. Si se pierden las vellosidades $\mathrm{y}$ la necrosis se extiende a la capa muscular puede no detectarse. ${ }^{(19)}$

- La I-FABP se expresa en todo el tracto gastrointestinal, con mayor concentración en el yeyuno, sin 
embargo, no puede determinar la localización de la necrosis ni por los niveles de esta proteína ni por el análisis de diferentes proteínas ligadoras de ácidos grasos. ${ }^{(9)}$

\section{CONCLUSIONES}

La presente revisión muestra que en la mayoría de los estudios tanto los niveles sanguíneos como urinarios de la I-FABP tienen una especificidad importante, asociado con sensibilidades variables para el diagnóstico de EAN, lo cual indica que es más útil para descartar la enfermedad que para diagnosticarla.

Sin embargo, varios estudios han demostrado que los niveles altos de la misma se relacionan con estadíos más severos de la enfermedad, tanto para determinar los casos que requieren cirugía como los casos con mayor probabilidad de muerte.

Además, parece haber cierta relación entre los niveles tanto sanguíneos como urinarios de esta proteína con el tamaño del intestino resecado durante cirugía, sin embargo, se requieren de estudios mejor diseñados para determinar esto.

\section{BIBLIOGRAFÍA}

1. Holman, R., Stoll, B., Curns, A. Necrotising enterocolitis hospitalisations among neonates in the United States. Paediatric and Perinatal Epidemiology. 2006; 20: 498-506.

2. Lanhella, F., Mangueira, L., Lopes, R., Vertoncini, A., Melo, R., \& Sbragia, L. Evaluation of the expression of I-FABP and $L$ $F A B P$ in a necrotizing enterocolitis model after the use of Lactobacillus acidophilus. Journal of Pediatric Surgery. 2014; 50: 543549 .

3. Guillet, R., Stoll, B., Cotton, C. Association of H2-blocker therapy and higher incidence of necrotizing enterocolitis in very low birth weight infants. Pediatrics. 2006; 117: 137142
4. Lin, P., Stoll, B. Necrotizing enterocolitis. Lancet. 2006; 268: 1271-83.

5. Ng, E., Poon, T., Lam, H., Cheung, H., Ma, T., Chan, K., Wong, R., Leung, K., Lam, M., Li, K., \& Ng, P. Gut-associated biomarkers L-FABP, I-FABP, and TFF3 and LIT Score for diagnosis of surgical necrotizing enterocolitis in preterm infants. Annals of Surgery. 2013; 258: 1111-1118.

6. Hintz, S., Kendrick, D., Stoll, B. Neurodevelopmental and growth outcomes of extremely low birth weight infants after necrotizing enterocolitis. Pediatrics. 2005; 115 : 696-703.

7. Benkoe, T., Mechtler, T., Weninger, M., Pones, M., Rebhandl, W., \& Kasper D. Serum levels of interleukin-8 and gut-associated biomarkers in diagnosing necrotizing enterocolitis in preterm infants. Journal of Pediatric Surgery. 2014; 49: 1446-1451.

8. Walsh, M., Kliegman, R. Necrotizing enterocolitis: treatment based on staging criteria. Pediatric Clinics of North America. 1986; 33: 179-201.

9. Heida, F., Hulscher, J., Schurink, M., Timmer, A., Kooi, E., Bos, A., Bruggink, J., Kasper, D., Pones, M., \& Benkoe, T. Intestinal fatty acid-binding protein levels in necrotizing enterocolitis correlate with extent of necrotic bowel: results from a multicenter study. Journal of Pediatric Surgery. 2014; 50: 1115-1118.

10. Reisinger, K., Kramer, B., Derikx, J. Noninvasive serum amyloid A measurement and plasma platelets for accurate prediction of surgical intervention in severe necrotizing enterocolitis. Public Library of Science One, 9. 2014

11. Dilli, D., Suna, S., Erol R., Ozkan H., Dumanli H., Dilmen U. Does abdominal sonography provide additional information over abdominal plain radiography for diagnosis of necrotizing enterocolitis in neonates?. Pediatric Surgical International. $2010 ; 3: 321-327$ 
12. Evennett, N., Alexander, N., Petrov, M., Pierro, A., \& Eaton, S. A systematic review of serologic tests in the diagnosis of necrotizing enterocolitis. Journal of Pediatric Surgery. 2009; 44: 2192-2201.

13. Sharma, R., Hudak, M. A clinical perspective of necrotizing enterocolitis: past, present, and future. Clinincs in Perinatology. 2013; 40: 27-51.

14. Aydemir, C., Dilli, D., Suna, S., Ozkan, H., Uras, N., Erdeve, O., \& Dilmen, U. Serum intestinal fatty acid binding protein level for early diagnosis and prediction of severity of necrotizing enterocolitis. Early Human Development. 2011; 87: 659-661.

15. Pelsers, M., Hermens, W., Glatz, J. Fatty acid-binding proteins as plasma markers of tissue injury. Clinica Chimica Acta. 2005; 352: 15-35.

16. Pelsers, M., Namiot, Z., Kisielewski, W. Intestinal-type and liver-type fatty acid binding protein in the intestine. Tissue distribution and clinical utility. Clinical Biochemistry. 2003; 36: 529-535.

17. Cronk, D., Houseworth, T., Cuadrado, D. Intestinal fatty acid binding protein for the detection of strangulated mechanical small bowel obstruction. Current Surgery. 2006; 63: 322-325.

18. Gregory, K., Winston, A., Yamamoto, H., Dawood, H., Fashemi, T., Fichorova, R., \& Van Marter, L. Elevated urinary iFABP predicts necrotizing enterocolitis within seven days prior to clinical onset. Journal of Pediatrics. 2014; 164: 1486-1488.

19. Evennett, N., Hall, N., Pierro, A., \& Eaton, S. Urinary intestinal fatty acid-binding protein concentration predicts extent of disease in necrotizing enterocolitis. Journal of Pediatric Surgery. 2009; 45: 735-740.

20. van de Poll, M., Derikx, J., Buurman, W. Liver manipulation causes hepatocyte injury and precedes systemic inflammation in pa- tients undergoing liver resection. World Journal of Surgery. 2007; 31: 2033-2038.

\section{CONFLICTO DE INTERÉS Y/O AGRADECIMIENTOS}

El autor declara que no existen conflictos de interés con la presente revisión bibliográfica. 